\title{
CHARACTERIZATION OF SEMINORMABILITY OF A TOPOLOGICAL ALGEBRA
}

\author{
PAMMY MANCHANDA
}

\begin{abstract}
A bstract. Let $\mathcal{A}$ be an algebra over a field $F$ and let $N$ be a norm on $F$. A seminorm (norm) on $\mathcal{A}$ associated with $N$ is defined. It is proved that if $(\mathcal{A}, \mathcal{J})$ is a proper topological algebra over a proper topological field $(F, T)$, then $T$ is defined by a norm $N$ and $\mathcal{J}$ is defined by a seminorm $\|\cdot\|$ associated with $N$ (a norm $\|\cdot\|$ associated with $N$ if $\mathcal{J}$ is Hausdorff) if and only if the following three conditions are satisfied.
\end{abstract}

(i) $(F, T)$ has a nonempty open bounded set.

(ii) $(F, T)$ has a nonzero topological nilpotent element.

(iii) $(\mathcal{A}, \mathcal{J})$ has a nonempty open bounded set.

\section{Introduction}

A. Kolmogorov [1] was the first to give necessary and sufficient conditions for a topological vector space (over real or complex numbers) to have its topology defined by a norm. Later G. Köthe [2] extended the result of Kolmogorov and gave necessary and sufficient conditions for a topological vector space (over real or complex numbers) to have its topology defined by a $p$-norm, $0<p \leq 1$. By a $p$-norm, $0<p \leq 1$ we mean a real valued function $\|\cdot\|$ defined on a vector space $V$ (over real or complex numbers) satisfying the following properties:

i) $\|x\| \geq 0$ for all $x \in V$ and $\|x\|=0$ if and only if $x=\theta$.

ii) $\|x+y\| \leq\|x\|+\|y\|$ for all $x, y \in V$

iii) $\|\lambda x\|=|\lambda|^{p}\|x\|$ for all $x \in V$ and scalar $\lambda$.

Received January 6, 1991; revised April 13, 1992. 
Here and subsequently $\theta$ will denote the zero vector. In [4] S. Singh defined a seminorm on a vector space $V$ associated with a norm $N$ defined on the scalar field $F$ of $V$. He gave necessary and sufficient conditions for a proper topological vector space $V$ over a proper topological field $F$ to have the topology on $F$ defined by a norm $N$ and the topology on $V$ defined by a seminorm $\|\cdot\|$ associated with $N$. In this note we define seminorm (norm) on an algebra $\mathcal{A}$ associated with a norm $N$ defined on the scalar field $F$ of $\mathcal{A}$ and find necessary and sufficient conditions for a proper topological algebra $\mathcal{A}$ over a proper topological field $F$ to have the topology on $F$ induced by a norm $N$ and topology on $\mathcal{A}$ induced by a seminorm (norm) associated with $N$. It is shown that if $(\mathcal{A}, \mathcal{J})$ is a topological algebra over a topological field $(F, T)$ such that the topology $\mathcal{J}$ on $\mathcal{A}$ is defined by a seminorm (treating $\mathcal{A}$ as a vector space over $F$ ) associated with a norm $N$ defining $T$, then the topology $\mathcal{J}$ on $\mathcal{A}$ is define by a seminorm (treating $\mathcal{A}$ as an algebra over $F$ ) associated with a norm $N^{\prime}$ defining $T$. Moreover, an example of a topological algebra $(\mathcal{A}, \mathcal{J})$ over a topological field $(F, T)$ is given, where $T$ is defined by a norm $N$ but $\mathcal{J}$ is not defined by any seminorm associated with $N$ or any other norm $N^{\prime}$ on $F$ defining the topology $T$. For the proof of the main theorem we use the techniques of Seth Warner [3].

\section{Normed Fields and Algebras}

In this section we give basic definitions and elementary properties of norms on fields and algebras.

Definition 1. Let $F$ be a field. By a norm on $F$ we mean a real valued function $N$ defined on $F$ such that

1) $N(\alpha) \geq 0$ for all $\alpha \in F$ and $N(\alpha)=0$ if and only if $\alpha=0$

2) $N(\alpha \beta) \leq N(\alpha) N(\beta)$ for all $\alpha, \beta \in F$.

3) $N(\alpha-\beta) \leq N(\alpha)+N(\beta)$ for all $\alpha, \beta \in F$.

A norm $N$ is said to be nontrivial if there exists a nonzero $\alpha \in F$ satisfying $0<N(\alpha)<1$, otherwise it is said to be a trivial norm, A norm $N$ is non 
archimedean if it satisfies the ultrametric inequality

$\left.3^{\prime}\right) N(\alpha-\beta) \leq \max \{N(\alpha), N(\beta)\}$ for all $\alpha, \beta \in F$.

A norm $N$ is said to be an absolute value if it satisfies the equality $\left.2^{\prime}\right) N(\alpha \beta)=N(\alpha) N(\beta)$ for all $\alpha, \beta \in F$.

Remark 1 . Every norm $N$ on a field $F$ gives rise to a topology defined by the metric $d(\alpha, \beta)=N(\alpha-\beta)$ for all $\alpha, \beta \in F$. Clearly the topology defined by a trivial norm is discrete.

Remark 2. If $F$ is a finite field and $N$ is a norm on $F$, then $N$ is a trivial norm. Because for each $\alpha \in F, \alpha \neq 0$ there exists a positive integer $n$ such that $\alpha^{n}=1$. Therefore

$$
1 \leq N(1)=N\left(\alpha^{n}\right) \leq N(\alpha)^{n}
$$

and hence $N(\alpha) \geq 1$.

In light of Remarks 1 and 2 the study of trivial norms on fields is not of much importance. Therefore from now onwards, by a norm on a field $F$ we always mean a non trivial norm on $F$. This will ensure that if $N$ is a norm on $F$ there always exists a nonzero element $\alpha$ in $F$ satisfying $0<N(\alpha)<1$.

Definition 2. Let $\mathcal{A}$ be an algebra over a filed $F$ and let $N$ be a norm on $F$. A seminorm on $\mathcal{A}$ associated with $N$ is a real valued function $\|\cdot\|$ defined on $A$ with the following properties:

1. $\|x\| \geq 0$ for all $x \in \mathcal{A},\|\theta\|=0$ and there exists at least one $x \in \mathcal{A}$ for which $\|x\| \neq 0$.

2. $\|\alpha x\| \leq N(\alpha)\|x\|$ for all $\alpha \in F, x \in \mathcal{A}$.

3. $\|x-y\| \leq\|x\|+\|y\|$ for all $x, y \in \mathcal{A}$.

4. $\|x y\| \leq\|x\|\|y\|$ for all $x, y \in \mathcal{A}$.

A seminorm $\|\cdot\|$ associated with $N$ is called a norm if

$$
\|x\|=0, x \in \mathcal{A} \text { if and only if } x=\theta .
$$

A seminorm (norm) $\|\cdot\|$ associated with $N$ is called non archimedean if it satisfies the ultrametric inequality 
$\left.3^{\prime}\right)\|x-y\| \leq \operatorname{Max}\{\|x\|,\|y\|\}$ for all $x, y \in \mathcal{A}$.

Remark 3. Let $F$ be a field with a norm $N$ defined on it. Let $\mathcal{A}$ be an algebra over $F$ and $\|\cdot\|$ be a seminorm on $\mathcal{A}$ associated with norm $N$, then one can check that for all $x, y \in \mathcal{A}$

i) $\|-x\|=\|x\|$;

ii) $\|x+y\| \leq\|x\|+\|y\|$.

In fact, (i) and (ii) together are equivalent to condition 2 in Definition 2.

Remark 4. Let $\mathcal{A}$ be an algebra over a field $F$. If $\|\cdot\|$ is a seminorm on $\mathcal{A}$ associated with an absolute value $N$ on $F$ then for every $\alpha \neq 0$ in $F$ and $x \in \mathcal{A}$

$$
\begin{aligned}
\|x\|=\left\|\alpha^{-1} \alpha x\right\| & \leq N\left(\alpha^{-1}\right)\|\alpha x\| \\
& \leq N\left(\alpha^{-1}\right) N(\alpha)\|x\| \\
& =N(1)\|x\| \\
& =\|x\|
\end{aligned}
$$

and hence $\|x\|=N\left(\alpha^{-1}\right)\|\alpha x\|$. Therefore $\|\alpha x\|=N(\alpha)\|x\|$. The equality

$$
\|\alpha x\|=N(\alpha)\|x\|
$$

also holds for $\alpha=0$ and every $x \in \mathcal{A}$. Therefore we have

$$
\|\alpha x\|=N(\alpha)\|x\| \text { for all } \alpha \in F, x \in \mathcal{A} \text {. }
$$

This shows that our definition of seminorm (norm) on an algebra associated with a norm on a scalar field coincides with the classical definition of seminorm (norm) defined over an absolute valued scalar field.

Remark 5. Let $F$ be a field with a norm $N$ defined on it. Let $\mathcal{A}$ be an algebra over $F$ and $\|\cdot\|$ be a seminorm on $\mathcal{A}$ associated with $N$. Then there exists a unique topology $\mathcal{J}$ on $\mathcal{A}$ defined by the pseudometric $d^{\prime}(x, y)=\|x-y\|$ for all $x, y \in \mathcal{A}$. This topology $\mathcal{J}$ is non discrete as justified below. There exists a nonzero element $x$ in $\mathcal{A}$ for which $\|x\| \neq 0$. Also there exists a nonzero $\alpha \in F$ 
for which $0<N(\alpha)<1$. Now for a given $\varepsilon>0$, there exists a positive integer $n$ such that

$$
N(\alpha)^{n}<\frac{\varepsilon}{\|x\|}
$$

Therefore

$$
\left\|\alpha^{n} x\right\| \leq N(\alpha)^{n}\|x\|<\varepsilon
$$

and hence every $\varepsilon$ neighbourhood of $\theta$ contains a nonzero element $\alpha^{n} x$ of $\mathcal{A}$ for some positive integer $n$. This shows that the topology $\mathcal{J}$ is non discrete.

Example 1 . Every field $F$ with a norm $N$ defined on it can be regarded as a normed algebra over itself or its subfield with norm $\|\cdot\|$ associated with $N$ defined by $\|x\|=N(x)$ for all $x \in F$.

Example 2. Let $F$ be any field with a non archimedean norm defined on it. The set $F[[X]]$ consisting of all power series in $X$ with coefficients from $F$ forms an algebra over $F$ with respect to the usual operations. It is straightforward to verify that the subcollection $\mathcal{A}$ of all those power series $f(X)=\sum_{n \geq 0} a_{n} X^{n}$ such that $\lim _{n} N\left(a_{n}\right)=0$ forms a subalgebra. The function $\|\cdot\|$ defined on $\mathcal{A}$ by

$$
\|f(X)\|=\sup _{n} N\left(a_{n}\right)
$$

is a non archimedean norm on $\mathcal{A}$ associated with $N$. Clearly the collection $F[X]$ of polynomials is a subalgebra of $\mathcal{A}$ and hence the restriction of above norm on $F[X]$ is also a norm on $F[X]$ associated with $N$.

Definition 3. Let $F$ be a field. A pair $(F, T)$ is said to be a topological field if $T$ is a topology on $F$ and the mappings $(\alpha, \beta) \rightarrow \alpha \pm \beta,(\alpha, \beta) \longrightarrow \alpha \beta$ and $\alpha \longrightarrow \alpha^{-1}(\alpha \neq 0)$ are continuous. If $T$ is neither discrete nor antidiscrete we say that $T$ is a proper field topology on $F$ and $(F, T)$ is a proper topological field.

Definition 4. Let $(\mathbb{F}, T)$ be a topological field and $\mathcal{A}$ be an algebra over $F$. A pair $(\mathcal{A}, \mathcal{J})$ is said to be a topological algebra over a topological field $(\mathbb{F}, T)$ 
if $\mathcal{J}$ is a topology on $\mathcal{A}$ and the mappings $(x, y) \longrightarrow x \pm y,(x, y) \longrightarrow x y$ and $(\alpha, x) \longrightarrow \alpha x$ for all $x, y \in \mathcal{A}, \alpha \in F$ are continuous. If $\mathcal{J}$ is neither discrete nor antidiscrete we say that $\mathcal{J}$ is a proper algebra topology on $\mathcal{A}$ and $(\mathcal{A}, \mathcal{J})$ is a proper topological algebra.

Remark 6. Let $F$ be a field with a norm $N$ defined on it. Let $\mathcal{A}$ be an algebra over $F$ and $\|\cdot\|$ be a seminorm (norm) on $\mathcal{A}$ associated with $N$. If $T$ and $\mathcal{J}$ are the topologies defined by $N$ and $\|\cdot\|$ on $F$ and $\mathcal{A}$ respectiviely, then it is well known that $(F, T)$ is a proper Hausdorff topological field and one can check as routine matter that $(\mathcal{A}, \mathcal{J})$ is a proper (Hausdorff) topological algebra over the topolgical field $(F, T)$.

Definition 5. Let $(\mathcal{A}, \mathcal{J})$ be a topological algebra over a topological field $(F, T)$. A subset $B$ of $F$ is said to be bounded if given any neighbourhood $U$ of 0 in $F$ there exists a neighbourhood $V$ of 0 in $F$ such that $V B \subseteq U$. Here $V B=\{\alpha \beta: \alpha \in V, \beta \in B\}$.

A subset $X$ of $\mathcal{A}$ is said to be bounded if given any neighbourhood $Y$ of $\theta$ in $\mathcal{A}$ there exists a neighbourhood $U$ of 0 in $F$ such that $U X \subseteq Y$. Here $U X=\{\alpha x: \alpha \in U, x \in X\}$. For the proof of the main theorem we need the following lemmas.

Lemma 1 . i) Let $(F, T)$ be a proper topological filed. Then the field $F$ cannot be bounded unless the topology $T$ defined on $F$ is discrete or antidiscrete.

ii) Let $(\mathcal{A}, \mathcal{J})$ be a topological algebra over a proper topological field $(F, T)$. Then the algebra $\mathcal{A}$ cannot be bounded unless the topology $\mathcal{J}$ defined on $\mathcal{A}$ is antidiscrete.

Proof. i) Let $F$ be bounded. Suppose $T$ is not discrete. Therefore every neighbourhood of zero must contain a nonzero element of $F$. Let $U$ be a given neighbourhood of zero. Since $F$ is bounded, there exists a neighbourhood $V$ of 0 in $F$ such that $V F \subseteq U$. Because $V$ contains a nonzero element say $\alpha$, we have

$$
1=\alpha \alpha^{-1} \in V F \subseteq U \text {. }
$$


This shows that 1 belongs to every neighbourhood of zero. In particular 1 belongs to the above neighbourhood $V$ of zero and hence $F \subseteq U$. Therefore $F=U$ and $F$ is the only neighbourhood of zero and hence $T$ is antidiscrete.

The proof for ii) similar.

Lemma 2. Let $(\mathcal{A}, \mathcal{J})$ be a topological algebra over a topological field $(F, T)$.

(i) If $A$ and $B$ are bounded subsets of $F$ then $A+B,-A$ and a $A$ for every $a \in F$ are also bounded. In particular $a+A$ for every $a \in F$ is also bounded.

(ii) If $X$ and $Y$ are bounded subsets of $\mathcal{A}$ then $X+Y$ and aX for every $a \in F$ are also bounded. In particular $x+X$ for every $x \in \mathcal{A}$ is also bounded.

Proof. Straightforward.

Lemma 3. Let $N$ be a norm on a field $F$ and $\|\cdot\|$ be a seminorm on an algebra $\mathcal{A}$ over $F$ associated with $N$.

i) $A$ subset $A \subseteq F$ is bounded if and only if there exists a real number $r>0$ such that $N(\alpha) \leq r$ for all $\alpha \in A$.

ii) A subset $X \subseteq \mathcal{A}$ is bounded if and only if there exists a real number $s>0$ such that $\|x\| \leq s$ for all $x \in X$.

Proof. Straightforward.

Lemma 4. Let $G$ be an additive abelian group and $v$ be a real function on $G$ taking values as either zero or integral powers of $d$, for some $d, 1<d \leq 2$, and $v(g+h) \leq d \operatorname{Max}\{v(g), v(h)\}$ for all $g, h \in G$. Then given any decomposition of $g$ in $G$ as $g=g_{1}+g_{2}+\cdots+g_{n}$, there exists a second decomposition of $g$ in $G$ as

$$
g=h_{1}+h_{2}+\cdots+h_{m}
$$

so that

$$
v\left(h_{1}\right)+\cdots+v\left(h_{m}\right) \leq v\left(g_{1}\right)+\cdots+v\left(g_{n}\right)
$$

and $v(g) \leq d v\left(h_{m}\right)$. 
Proof. First we prove that there is a decomposition of $g$ in $G$ as $g=$ $h_{1}+h_{2}+\cdots+h_{m}$ which satisfies the following

$$
\begin{aligned}
& v\left(h_{1}\right)+v\left(h_{2}\right)+\cdots+v\left(h_{m}\right) \leq v\left(g_{1}\right)+\cdots+v\left(g_{n}\right) \\
& \text { and } v\left(h_{i}+h_{j}\right)>v\left(h_{i}\right)+v\left(h_{j}\right) \text { for all } 1 \leq i, \quad j \leq m, j \neq j
\end{aligned}
$$

If the given decomposition $g=g_{1}+\cdots+g_{n}$ does not satisfy (2) then there exist $g_{i}$ and $g_{j}, 1 \leq i, j \leq n, i \neq j$ such that

$$
v\left(g_{i}+g_{j}\right) \leq v\left(g_{i}\right)+v\left(g_{j}\right) .
$$

Then we do not increase the sum on left of (1) if we replace $v\left(g_{i}\right)+v\left(g_{j}\right)$ by $v\left(g_{i}+g_{j}\right)$. We combine $g_{i}$ and $g_{j}$ into a single term in the given decomposition of $g$ and get a new decomposition of $g$ as $g=g_{1}+\cdots+g_{i-1}+g_{i+1}+\cdots+g_{j-1}+$ $g_{j+1}+\cdots+g_{n}+\left(g_{i}+g_{j}\right)$. This decreases the number of terms in the given decomposition by one. If the new decomposition does not satisfy (2) we repeat the above process which again reduces the number of terms by one. Continuing in this way, after a finite number of steps we get a decomposition $g=\sum_{j=1}^{m} h_{j}$ of $g$ satisfying (1) and (2) or a single term. Moreover we may assume that

$$
v\left(h_{1}\right) \leq v\left(h_{2}\right) \leq \cdots \leq v\left(h_{m}\right)
$$

If $v\left(h_{i}\right)=v\left(h_{i+1}\right)$ for some $i=1,2, \ldots, m-1$, then

$$
\begin{aligned}
v\left(h_{i}+h_{i+1}\right) & \leq d \operatorname{Max}\left\{v\left(h_{i}\right), v\left(h_{i+1}\right)\right\} \\
& =d v\left(h_{i}\right) \\
& \leq v\left(h_{i}\right)+v\left(h_{i+1}\right)
\end{aligned}
$$

a contradiction to (2).

Therefore $v\left(h_{i}\right)<v\left(h_{i+1}\right)$ for all $i=1,2, \ldots, m-1$. Since $v$ takes values as integral powers of $d$, we get

$$
d v\left(h_{i}\right) \leq v\left(h_{i+1}\right) \text { for } i=1,2, \ldots, m-1 \text {. }
$$


Now we show by induction on $t$, that

$$
v\left(h_{1}+h_{2}+\cdots+h_{t}\right) \leq d v\left(h_{t}\right) \text { for } t=1,2, \ldots, m .
$$

When $t=1$, the result clearly holds.

Suppose it holds for all $t, 1 \leq t \leq k-1 \leq m-1$. Then

$$
\begin{aligned}
v\left(h_{1}+h_{2}+\cdots+h_{k}\right) & \leq d \operatorname{Max}\left\{v\left(h_{1}+\cdots+h_{k-1}\right), v\left(h_{k}\right)\right\} \\
& \leq d \operatorname{Max}\left\{d v\left(h_{k-1}\right), v\left(h_{k}\right)\right\} \\
& =d v\left(h_{k}\right)
\end{aligned}
$$

and hence by induction it is true for all $t$.

Taking $t=m$, we get

$$
v\left(h_{1}+h_{2}+\cdots+h_{m}\right) \leq d v\left(h_{m}\right)
$$

that is $v(g) \leq d v\left(h_{m}\right)$.

This completes the proof of the lemma.

\section{Characterization of seminorm topology on an algebra}

In this section we give necessary and sufficient conditions for a proper topological algebra $(\mathcal{A}, \mathcal{J})$ over a proper topological field $(F, T)$ to have the topology $T$ on $F$ defined by a norm $N$ and the topology $\mathcal{J}$ on $\mathcal{A}$ defined by a seminorm (norm in case $\mathcal{J}$ is Hausdorff) $\|\cdot\|$ associated with $N$. For this we use the techniques of Seth Warner [3].

Theorem 1. Let $(F, T)$ be a proper topological field and $(\mathcal{A}, \mathcal{J})$ be a proper topological algebra over $(F, T)$. Then $T$ is defined be a norm $N$ on $F$ and $\mathcal{J}$ is defined by a seminorm (norm in case $\mathcal{J}$ is Hausdorff) $\|\cdot\|$ on $\mathcal{A}$ associated with $N$ if and only if

(i) $(F, T)$ has a nonempty open bounded set.

(ii) $(F, T)$ has a nonzero topological nilpotent element.

(iii) $(\mathcal{A}, \mathcal{J})$ has a nonempty open bounded set. 
Proof. Let $T$ be defined by some norm $N$ on $F$ and $\mathcal{J}$ by some seminorm $\|\cdot\|$ on $\mathcal{A}$ associated with $N$. Take

$$
B=\{\alpha \in F: N(\alpha)<1\}, X=\{x \in \mathcal{A}:\|x\|<1\} .
$$

Clearly $B$ is nonempty and $X$ is nonempty (because $0 \in B$ and $\theta \in X$ ) and are open. By lemma $3, B$ is bounded in $F$ and $X$ is bounded in $\mathcal{A}$. Also there exists a nonzero element $a \in F$ satisfying $0<N(a)<1$. This ' $a$ ' is a topological nilpotent element in $F$. By Remark 5 , for a given $\varepsilon>0$ there exists a nonzero element $x$ in $\mathcal{A}$ satisfying $0<\|x\|<\varepsilon$, therefore topology $\mathcal{J}$ is proper. Also note that if $\|\cdot\|$ is a norm then $\mathcal{J}$ is Hausdorff.

We now prove the converse. Let $B$ be an open nonempty bounded subset of $F$. If $0 \notin B$, we choose some element $b \in B$ and replace $B$ by $B^{\prime}=B-b$. The set $B^{\prime}$ is again open and using lemma $2, B^{i}$ is bounded which contains $0=b-b$. Replace $B^{\prime}$ by $B^{\prime \prime}=B^{\prime} \cap\left(-B^{\prime}\right)$. Then $B^{\prime \prime}$ is a symmetric, open bounded set containing 0 . Therefore we can assume without loss of generality that $B$ is a symmetric, open, bounded set containing 0 . We now prove that the collection $\{b B\}$ as $b$ varies over nonzero elements of $F$ forms a neighbourhood base of 0 . Since $(F, T)$ is a topological field, for each $b \neq 0, b B$ is a neighbourhood of 0 . Let $P$ be any neighbourhood of 0 . Since $B$ is bounded there exists a neighbourhood $D$ of 0 such that $D B \subseteq P$. As $T$ is proper there exists a nonzero element $b$ in $D$. Therefore $b B \subseteq P$.

By continuity of multiplication in $F$ there exists a nonzero element $b$ in $F$ such that $b B . b B \subseteq B$. Put $B_{1}=b^{2} B$, then $B_{1}$ is again a symmetric, bounded neighbourhood of 0 . In addition

$$
B_{1} \cdot B_{1}=b^{4} B B \subseteq b^{2} B=B_{1}
$$

and the collection $\left\{b B_{1}\right\}$ where $b$ varies over nonzero elements of $F$ forms a neighbourhood base of zero. Using continuity of multiplication and addition, we can find a neighbourhood $C$ of 0 satisfying $C \subseteq B_{1}$ and

$$
C B_{1}+C B_{1}+C B_{1}, \subseteq B_{1} \text {. }
$$


Let ' $a$ ' be a topological nilpotent element of $F$. There exists a positive integer $k$ such that $a^{k} \in C$. Replacing a by $a^{k}$ we can assume that $a \in C$ and hence

$$
a B_{1}+a B_{1}+a B_{1} \subseteq B_{1}
$$

We now prove the following:

$$
\cdots a^{2} B_{1} \subset a B_{1} \subset B_{1} \subset a^{-1} B_{1} \subset a^{-2} B_{1} \subset \cdots
$$

is a strictly ascending sequence.

$$
\begin{aligned}
& F=\bigcup_{n=1}^{\infty} a^{-n} B_{1} . \\
& \bigcap_{n=1}^{\infty} a^{n} B_{1}=\{0\} .
\end{aligned}
$$

The collection $\left\{a^{n} B_{1}\right\}, n=0,1,2, \ldots$ forms a neighbourhood base of 0 in $F$.(6) First we prove (4). If $\alpha \in F$, then because $a^{n} \alpha \rightarrow 0$ in $F$ and $B_{1}$ is a neighbourhood of 0 , there exists a positive integer $n$ such that $a^{n} \alpha \in B_{1}$. Therefore $\alpha \in a^{-n} B_{1}$ and hence

$$
F=\bigcup_{n=1}^{\infty} a^{-n} B_{1} .
$$

Next we prove (3). First we observe that $a \in B_{1}$ and $B_{1} \cdot B_{1} \subseteq B_{1}$, therefore $a B_{1} \subseteq B_{1}$. From this it follows that $a^{n+1} B_{1} \subseteq a^{n} B_{1}$ for each integer $n$. Suppose there exists an integer $m$ such that $a^{m+1} B_{1}=a^{m} B_{1}$. Because $a \in B_{1}$, therefore $a^{m}$. $a \in a^{m} B_{1}=a^{m+1} B_{1}$ and hence $a^{m+1} \in a^{m+1} B_{1}$ which implies $1 \in B_{1}$. Now

$$
a^{m} \cdot 1 \in a^{m} B_{1}=a^{m+1} B_{1}
$$

therefore $a^{-1} \in B_{1}$ which implies that $a^{-n} \in B_{1}$ for every positive integer $n$. Therefore $F=\bigcup_{n=1}^{\infty} a^{-n} B_{1} \subseteq B_{1}$, a contradiction, because $B_{1}$ is bounded but by lemma 1, F cannot be bounded. Therefore $a^{n+1} B_{1} \neq a^{n} B_{1}$ for every integer 
$n$. Now we prove that $\bigcap_{n=1}^{\infty} a^{n} B_{1}=(0)$. Suppose there exists a nonzero element $a \in \bigcap_{n=1}^{\infty} a^{n} B_{1}$. Since $F=\bigcup_{n=1}^{\infty} a^{-n} B_{1}$, there exists a positive integer $m$ such that $a^{m} \alpha^{-1} \in B_{1}$. Also $\alpha \in a^{m+1} B_{1}$ that is, $\alpha a^{-m-1} \in B_{1}$. Since $B_{1} \cdot B_{1} \subseteq B_{1}$, $\left(a^{m} \alpha^{-1}\right)\left(\alpha a^{-m-1}\right)=a^{-1} \in B_{1}$ which gives a contradiction as in the proof of (3). To prove (6), let $P$ be a given neighbourhood of 0 in $F$. There exists a neighbourhood $Q$ of 0 such that $Q B_{1} \subseteq P$ (because $B_{1}$ is bounded). Because $a^{n} \rightarrow 0$ there exists an integer $n \geq 0$ such that $a^{n} \in Q$ and hence $a^{n} B_{1} \subseteq P$. This shows that the collection $\left\{a^{n} B_{1}\right\}, n=0,1,2, \ldots$ forms a neighbourhood base of 0 in $F$.

Let $U$ be a given nonempty open bounded set in $\mathcal{A}$. As before, we can assume without loss of generality that $U$ is a symmetric, open, bounded subset of $\mathcal{A}$ containing $\theta$.

Now we claim that the collection $\left\{a^{n} U\right\} n=0,1,2, \ldots$ forms a neighbourhood base at $\theta$. By continuity of scalar multiplication, $a^{n} U$ is a neighbourhood of $\theta$ for each $n \geq 0$. Let $V$ be any neighbourhood of $\theta$. Then by boundedness of $U$ there exists a neighbourhood $P$ of 0 in $F$ such that $P U \subseteq V$. As ' $a$ ' is a topological nilpotent element of $F, a^{n} \in P$ for some $n$ and hence $a^{n} U \subseteq V$. Therefore the collection $\left\{a^{n} U\right\}, n=0,1,2, \ldots$ forms a neighbourhood base of $\theta$ in $\mathcal{A}$. Next, using continuity of multiplication and scalar multiplication in $\mathcal{A}$ we can find non negative integers $r$ and $s$ such that

$$
a^{r} U \cdot a^{r} U \subseteq U \text { and } a^{s} B_{1} \cdot a^{r} U \subseteq U
$$

Put $t=r+s$. Then

$$
a^{2 r} U \cdot U \subseteq U \text { and } a^{t} B_{1} U \subseteq U
$$

Put $U_{1}=a^{2 r} U$ and $B_{2}=a^{t} B_{1}$.

Then $U_{1} U_{1}=a^{2 r} U \cdot a^{2 r} U \subseteq a^{2 r} U=U_{1}$,

$$
\begin{aligned}
& B_{2} U_{1}=a^{t} B_{1} a^{2 r} U \subseteq a^{2 r} U=U_{1}, \\
& B_{2} B_{2}=a^{t} B_{1} \cdot a^{t} B_{1}=a^{2 t} B_{1} \cdot B_{1} \subseteq a^{2 t} B_{1} \subseteq a^{t} B_{1}=B_{2},
\end{aligned}
$$


and

$$
\begin{aligned}
a B_{2}+a B_{2}+a B_{2} & =a^{t+1} B_{1}+a^{t+1} B_{1}+a^{t+1} B_{1} \\
& =a^{t}\left(a B_{1}+a B_{1}+a B_{1}\right) \\
& \subseteq a^{t} B_{1} \\
& =B_{2}
\end{aligned}
$$

This $B_{2}$ will satisfy (3)-(6) if we replace $B_{1}$ by $B_{2}$. From this it follows that there exists a pair $(B, U)$ and a topological nilpotent element ' $a$ ' in $F$, where $B$ is a symmetric bounded neighbourhood of 0 in $\mathbb{F}$ and $U$ is a symmetric, bounded neighbourhood of $\theta$ in $\mathcal{A}$ satisfying the following:

1) $B B \subseteq B$

2) $a B+a B+a B \subseteq B$.

3) $\cdots a^{2} B \subset a B \subset B \subset a^{-1} B \subset \cdots$ is a strictly ascending sequence.

4) $F=\bigcup_{n=1}^{\infty} a^{-n} B$.

5) $\bigcap_{n=1}^{\infty} a^{n} B=(0)$

6) $\left\{a^{n} B\right\}, n=0,1,2, \ldots$ form a neighbourhood base of 0 in $F$.

7) $B U \subseteq U$ and $U U \subseteq U$.

8) $a U+a U+a U \subseteq U$.

9) $\cdots a^{2} U \subset a U \subset U \subset a^{-1} U \subset a^{-2} U \ldots$ is a strictly ascending sequence.

10) $\mathcal{A}=\bigcup_{n=1}^{\infty} a^{-n} U$.

11) $\bigcap_{n=1}^{\infty} a^{n} U=\{\theta\}$, if $\mathcal{J}$ is Hausdorff.

12) $\left\{a^{n} U\right\}, n=0,1,2, \ldots$ form a neighbourhood base of $\theta$ in $\mathcal{A}$.

To get a norm $N$ that generates the given topology $T$ on $\mathbb{F}$, we first define a function $f$ on $F$ as follows:

$$
f(\alpha)= \begin{cases}0 & \text { if } \alpha=0 \\ 2^{-n} & \text { if } \alpha \in a^{n} B \text { but } \alpha \notin a^{n+1} B\end{cases}
$$

(Note that from properties (3)-(5) of $B$ above, such an $n$ must exist and is unique). 
This $f$ takes values either 0 or integral powers of 2 and satisfies the following

(i) $f(\alpha)=0$ if and only if $\alpha=0$

(ii) $f(\alpha-\beta) \leq 2 \operatorname{Max}\{f(\alpha), f(\beta)\}$ for all $\alpha, \beta \in F$

(iii) $f(\alpha \beta) \leq f(\alpha) f(\beta)$ for all $\alpha, \beta \in F$

$N(\alpha)=\inf \sum_{i=1}^{p} f\left(\alpha_{i}\right)$, where the infimum is taken over all finite sums of $\alpha$ as $\alpha=\alpha_{1}+\alpha_{2}+\ldots+\alpha_{p}$ in $F$. One can check that $N$ is a norm on $F$. To prove that this $N$ induces the given topology $T$ on $F$ we first prove that $\frac{1}{2} f(\alpha) \leq N(\alpha) \leq$ $f(\alpha)$ for all $\alpha \in F$. The inequality $N(\alpha) \leq f(\alpha)$ follows from the definition of $N$. To prove $\frac{1}{2} f(\alpha) \leq N(\alpha)$ for all $\alpha \in F$, let $\alpha=\alpha_{1}+\alpha_{2}+\ldots+\alpha_{p}$ be a decomposition of $\alpha$ as a finite sum in $F$. By lemma 4 there exists a decomposition of $\alpha$.

$\alpha=\beta_{1}+\beta_{2}+\ldots+\beta_{q}$ as a finite sum in $F$ such that $f\left(\beta_{1}\right)+\ldots+f\left(\beta_{q}\right) \leq$ $f\left(\alpha_{1}\right)+\ldots+f\left(\alpha_{p}\right)$ and $f(\alpha) \leq 2 f\left(\beta_{q}\right)$.

Therefore $f(\alpha) \leq 2 \sum_{j=1}^{q} f\left(\beta_{j}\right) \leq 2 \sum_{i=1}^{p} f\left(\alpha_{i}\right)$ Hence $f(\alpha) \leq 2 N(\alpha)$ that is $\frac{1}{2} f(\alpha) \leq N(\alpha)$. From this inequality it follows that

$$
a^{n+1} B \subseteq\left\{\alpha \in F / N(\alpha)<\left(\frac{1}{2}\right)^{n}\right\} \subseteq a^{n} B
$$

Therefore $N$ induces the given topology $T$ on $F$.

Our next aim is to define a seminorm $\|\cdot\|$ on $\mathcal{A}$ associated with $N$ which induces the given topology $\mathcal{J}$ on $\mathcal{A}$. For this, first we define a function $g$ on $\mathcal{A}$ as follows:

$$
g(x)= \begin{cases}0 & \text { if } x \in \bigcap_{n=1}^{\infty} a^{n} U \\ 2^{-n} & \text { if there exists an integer } n \text { such that } \\ & x \in a^{n} U \text { but } x \notin a^{n+1} U\end{cases}
$$

(Note that if $\mathcal{J}$ is Hausdorff, then $\bigcap_{n=1}^{\infty} a^{n} U=(\theta)$ and therefore $g(x)=0$ if and only if $x=\theta$ ). This $g$ takes values either 0 or integral powers of 2 and satisfies the following

i) $g(\theta)=0$, there exists at least one $x$ in $\mathcal{A}$ such that $g(x) \neq 0$ and if $\mathcal{J}$ is Hausdorff, then $g(x)=0$ iff $x=\theta$. 
ii) $g(x+y) \leq 2 \operatorname{Max}\{g(x), g(y)\}$,

iii) $g(\alpha x) \leq f(\alpha) g(x)$,

iv) $g(x y) \leq g(x) g(y)$,

for all $x, y \in \mathcal{A}$ and $\alpha \in F$.

We now define a function $\|\cdot\|$ on $\mathcal{A}$ as $\|X\|=\inf \sum_{i=1}^{p} g\left(x_{i}\right)$, where the infimum is taken over all finite sums of $x$ as $x=x_{1}+x_{2}+\cdots+x_{p}$.

One can check, that $\|\cdot\|$ is a seminorm (norm if $\mathcal{J}$ is Hausdorff) on $\mathcal{A}$ associated with $N$. As in the case of $N,\|\cdot\|$ satisfies $\frac{1}{2} g(x) \leq\|x\| \leq g(x)$ for all $x \in \mathcal{A}$. From this inequality it follows that

$$
a^{n+1} U \subseteq\left\{x \in \mathcal{A} /\|x\|<(1 / 2)^{n}\right\} \subseteq a^{n} U
$$

Therefore $\|\cdot\|$ defines the given topology $\mathcal{J}$ on $\mathcal{A}$. This completes the proof of the theorem.

As an application of Theorem 1 we have the following theorem.

Theorem 2. Let $(\mathcal{A}, \mathcal{J})$ be a topological algebra over a topological field $(F, T)$. Let the topology $T$ on $F$ be defined by a norm $N$ and the topology $\mathcal{J}$ on $\mathcal{A}$ be defined by a seminorm $\|\cdot\|$, regarding $\mathcal{A}$ as a vector space over $F$ associated with $N$ (that is $\|\cdot\|$ is a real valued function on $\mathcal{A}$ satifying (1)-(3) of definition 2). Then there exists a norm $N^{\prime}$ on $F$ and a seminorm $\|\cdot\|^{\prime}$ on $\mathcal{A}$ associated with $N^{\prime}$ such that $T$ is defined by $N^{\prime}$ and $\mathcal{J}$ is defined by $\|\cdot\|^{\prime}$.

Proof. Take $B=\{\alpha \in F: N(\alpha)<1\}, X=\{x \in \mathcal{A}:\|x\|<1\}$ One can check that $B$ and $X$ are nonempty, open bouonded subsets of $F$ and $\mathcal{A}$ respectively. Also by assumption on $N$ there exists a nonzero element $a \in F$ satisfying $0<N(a)<1$. This ' $\mathrm{a}$ ' is a topological nilpotent element in $F$. Using converse of Theorem 1, the existence of desired $N^{\prime}$ and $\|\cdot\|^{\prime}$ follows.

Finally we given an example of a topological algebra whose topology is not defined by a seminorm.

Example 3. Let $F$ be a field with an absolute value $N$ defined on it. Let $\mathcal{A}=F[X]$ be the algebra of polynomials with coefficients from $F$. Let 
$\wedge=\left\{\varepsilon: \varepsilon=\left\langle\varepsilon_{0}, \varepsilon_{1}, \ldots>\right.\right.$ is a sequence of positive real numbers $\}$. For each $\varepsilon \in \wedge$, we take $U_{\varepsilon}=\left\{f(X)=\sum_{i \geq 0} a_{i} X^{i} \in \mathcal{A}: N\left(a_{i}\right)<\varepsilon_{i}\right.$ for all $\left.i\right\}$. There exists a unique ring topology $\mathcal{J}$ on $\mathcal{A}$ for which the collection $\left\{U_{\varepsilon}: \varepsilon \in \wedge\right\}$ froms a neighbourhood base of zero. Because $U_{\varepsilon} \cap F=\left\{a_{0} \in F: N\left(a_{0}\right)<\varepsilon_{0}\right\}$. Therefore $U_{\varepsilon} \cap F$ is clearly a neighbourhood of zero in $F$ with respect to the topology defined by $N$. Hence if $T$ is the relative topology of $\mathcal{J}$ on $F$ then $T$ is defined by the absolute value $N$. Hence

i) $(F, T)$ has a nonempty open bounded set.

ii) $(F, T)$ has a nonzero topological nilpotent element. We now claim that $(\mathcal{A}, \mathcal{J})$ does not have a nonempty open bounded set. For this it is sufficient to prove that given any $U_{\varepsilon}, \varepsilon \in \wedge$ there exists $a U_{\delta}, \delta \in \wedge$ such that for all nonzero $\alpha$ in $F, \alpha U \varepsilon \nsubseteq U_{\delta}$. Let $\varepsilon=<\varepsilon_{0}, \varepsilon_{1}, \ldots>\in \wedge$ be given. Choose an element $a \in F$, satisfying $0<N(a)<1$. Take $\delta=\left\langle\delta_{0}, \delta_{1}, \ldots>\right.$, where $\delta_{i}=$ $\varepsilon_{i} N(a)^{i}$. Suppose $\alpha$ is a nonzero element of $F$. There exist positive integers $m$ and $k_{m}$ satisfying $N(\alpha)>N(a)^{m-1}$ and $N(a)^{k_{m}}<\varepsilon_{m}<N(a)^{k_{m}-1}$.

Now clearly $a^{k_{m}} X^{m} \in U_{\varepsilon}$ but $\alpha a^{k_{m}} X^{m} \notin U_{\delta}$ because

$$
\begin{aligned}
N\left(\alpha a^{k_{m}}\right) & =N(\alpha) N(a)^{k_{m}} \\
& >N(a)^{m-1} N(a)^{k_{m}} \\
& =N(a)^{k_{m}-1+m} \\
& >N(a)^{m} \varepsilon_{m} \\
& =\delta_{m} .
\end{aligned}
$$

Therefore $\alpha U_{\varepsilon} \nsubseteq U_{\delta}$.

Now the topology $T$ on $F$ is defined by absolute value $N$, but by Theorem 1 the topology $\mathcal{J}$ on $\mathcal{A}$ is not defined by any seminorm associated with $N$ or with any other norm $N^{\prime}$ on $F$ which defines the topology $T$ on $F$.

\section{Acknowledgement}

The author is thankful to Dr. Surjit Singh for his constant guidance and advice and to the referee for his valuable comments. 


\section{References}

[1] Kolmogorov, A. "Zur Normierbarkeit eines allgemeinen topologische Raumes", Studia Math., 5 (1934), 29-33.

[2] Köthe G. "Topological vector spaces", Springer Verlag, (1969).

[3] Seth Warner, "Normability of certain topological rings", Proc. Amer. Math. Soc., 33 (1972), 423-427.

[4] S. Singh, "Pseudovaluation and Pseudonorm", Rend. Sem. Mat. Univ. Padova, 51 (1974), $1-14$.

Department of Mathematics Guru Nanak dev University Amritsar-143005 (India). 